\title{
Tuning the pore size from micro- to meso-porous in thermally stable aluminophosphates
}

\author{
Saúl Cabrera, ${ }^{a}$ Jamal El Haskouri, ${ }^{a}$ Carmen Guillem, ${ }^{a}$ Aurelio Beltrán-Porter ${ }^{a}$ Daniel Beltrán-Porter, ${ }^{a}$ \\ Sagrario Mendioroz, ${ }^{b}$ M. Dolores Marcos $* a$ and Pedro Amorós*a \\ a Institut de Ciència dels Materials de La Universitat de València (ICMUV), Dr. Moliner 50, 46100-Burjassot \\ (València), Spain.E-mail: loles.marcos@uv.es; pedro.amoros@uv.es \\ ${ }^{b}$ Instituto de Catálisis y Petroleoquímica, Campus Universidad Autónoma, Cantoblanco, 28049-Madrid, Spain
}

Received (in Bristol, UK) 16th December 1998, Accepted 12th January 1999

Thermally stable porous aluminophosphates (ICMUV-3) with $\mathrm{P} / \mathrm{Al}$ molar ratios in the range $0.15 \leqslant \mathrm{P} / \mathrm{Al} \leqslant 0.75$ and showing continuously adjustable pore sizes from 13 to $37 \AA$ have been prepared through a surfactant-assisted procedure without changing the surfactant length and/or addition of organic expansors.

Since the discovery in 1982 of three-dimensional aluminium phosphates (ALPOs), ${ }^{1}$ their chemistry has aroused great interest mainly due to the attainment of microporous structures showing a broad range of physicochemical properties of potential application in fields such as catalysis or molecular sieving. ${ }^{2,3}$ Control of pore geometry and diameter are key to many of these applications, especially in the case of those that rely on size or shape selectivity and ready access to pore system. The synthesis of ALPO molecular sieves, as for zeolites, involves the use of organic agents as 'templates'. ${ }^{4}$ In this sense, the synthetic strategy developed by Mobil scientists to obtain mesoporous silicas by use of micellar aggregates of surfactants as 'templates', 5 was also seen as very promising for the preparation of aluminophosphates. Thus, Stucky and coworkers ${ }^{6}$ were the first to extend the surfactant templating strategy to the synthesis of non-silica-based mesostructures, mainly metal oxides. However, in the case of the ALPO system this method has not been as fruitful as for silica-based materials. Although lamellar ${ }^{7}$ and hexagonal MCM-41-type mesostructured ALPOs ${ }^{8,9}$ have been prepared through surfactant-assisted synthetic strategies, the preparation of the corresponding mesoporous materials was not possible because their mesostructure collapsed upon calcination during surfactant removal. More recently, the preparation of two thermally stable mesoporous ALPOs using cationic surfactants has been described: Kevan and coworkers ${ }^{10,11}$ have reported the synthesis of non-ideal mesoporous aluminophosphates $(0.16 \leqslant \mathrm{P} / \mathrm{Al} \leqslant 0.60)$ with a constant pore size around 35 $\AA$, and Kuroda and coworkers ${ }^{12,13}$ have described the isolation of mesoporous ALPO's showing pore diameters modulated in the range 18-39 $\AA$ by changing the chain lengths of the surfactants and using organic cosurfactants as pore expansors.

We report here, a novel method to prepare thermally stable mesoporous aluminophosphates, denoted ICMUV-3, allowing a continuous adjustment of the pore sizes simply by regulation of both the P/Al molar ratio and the water content in the mothersolution.

Mesoporous ICMUV-3 materials have been prepared using cetyltrimethylammonium bromide $(\mathrm{CTABr})$ as surfactant structural directing agent in an aqueous TEA (triethanolamine) medium with a molar ratio in the starting solution $\mathrm{Al}: x \mathrm{P}: 0.3 \mathrm{C}$ TABr: 3.8TEA : $y \mathrm{H}_{2} \mathrm{O},(x=0.12-1.5, y=131-185)$. In a typical sample preparation, $\mathrm{Al}\left(\mathrm{OBu}^{\mathrm{s}}\right)_{3}(5.1 \mathrm{ml}, 0.02 \mathrm{~mol})$ was slowly added to liquid TEA $(10 \mathrm{ml} ; 0.075 \mathrm{~mol})$ and heated at $160{ }^{\circ} \mathrm{C}$. After $30 \mathrm{~min}$, the solution was cooled at $120^{\circ} \mathrm{C}$ and 2.1 $\mathrm{g}$ of CTABr $(0.006 \mathrm{~mol})$ added. Then, it was cooled at room temperature and mixed with $43 \mathrm{ml}(2.4 \mathrm{~mol})$ of distilled water. The resulting solution $\left(\mathrm{pH}_{\mathrm{i}}=10-11\right)$ was then combined with a given amount of diluted phosphoric acid $(0.55 \mathrm{~mol}$ in $10 \mathrm{ml}$ of water) under vigorous stirring $\left(\mathrm{pH}_{\mathrm{f}}=8-9\right)$, and the formation of a white solid was observed. This mesostructured solid was washed with warm water and air dried. In order to obtain the corresponding mesoporous material, the surfactant was thermally removed by stepped treatments under flowing air atmosphere $\left(2 \mathrm{~h}\right.$ at $300{ }^{\circ} \mathrm{C}, 2 \mathrm{~h}$ at $400{ }^{\circ} \mathrm{C}$ and finally at $500^{\circ} \mathrm{C}$ during $2 \mathrm{~h}$ ). All the samples were analyzed and characterized by X-ray powder diffraction techniques (Seifert 3000TT diffractometer using $\mathrm{Cu}-\mathrm{K} \alpha$ radiation), TEM (Philips $\mathrm{CM} 10$ instrument operated at $120 \mathrm{kV}$ ), electron probe microanalysis EPMA (Philips SEM-515 instrument), $\mathrm{N}_{2}$ adsorption-desorption isotherms (Micromeritics ASAP2010 analyzer) and ${ }^{27} \mathrm{Al}$ MAS NMR (Varian Unity-300 spectrometer operating at 300 MHz.

EPMA analysis shows that each ICMUV-3 mesoporous sample has a constant and well defined composition, showing in each case a homogeneous distribution of phosphorus atoms in the framework. Hence, monophasic character of the solids can be assumed. In all cases, ICMUV-3 solids have a non-ideal ALPO stoichiometry (P/Al < 1). ${ }^{27} \mathrm{Al}$ MAS NMR spectroscopy for as-synthesized and calcined ICMUV-3 materials show two resonance signals at $\delta c a .0$ and 50 . These signals are indicative of six- and four-coordinate metal centers, respectively. A relative intensity increase of the signal assigned to the fourcoordinate $\mathrm{Al}$ centers is observed as the P/Al ratio increases both in mesostructured and mesoporous solids. Also, an increase of that signal is observed after calcination.

Selected XRD patterns of as-synthesized and mesoporous ICMUV-3 samples are shown in Fig. 1. All solids present at least one very intense peak at low $2 \theta$ values $\left(d_{100}=28-57 \AA\right)$. It should be remarked that the position of this peak closely follows the variation of the P/Al molar ratio in the solids: the

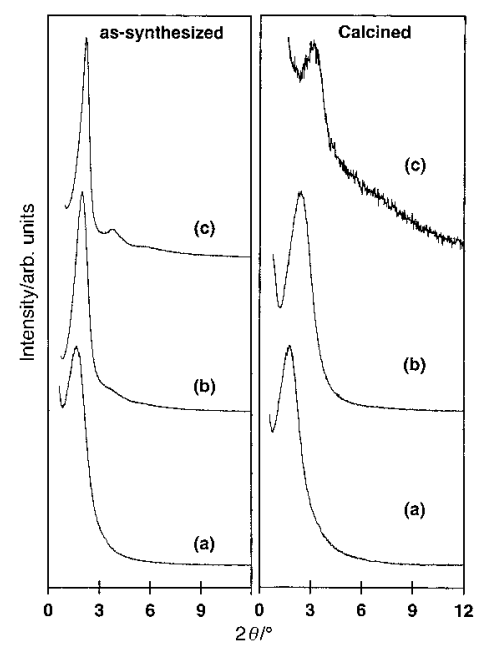

Fig. 1 Representative X-ray powder diffraction patterns of both assynthesized and porous ICMUV-3 solids; (a) $\mathrm{P} / \mathrm{Al}=0.17$, (b) $\mathrm{P} / \mathrm{Al}=0.55$, (c) $\mathrm{P} / \mathrm{Al}=0.74$. 
as-synthesized
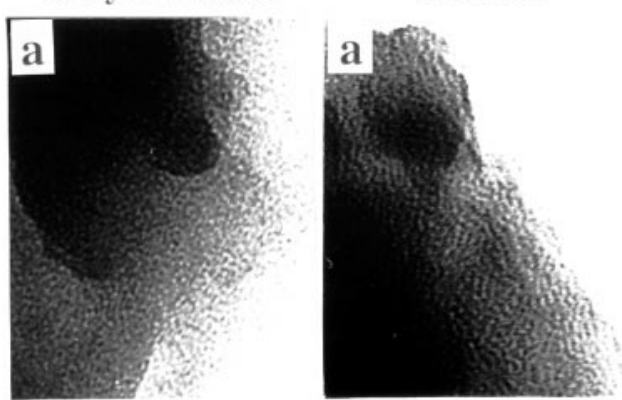

b

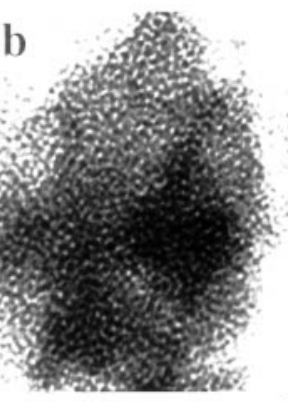

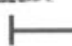

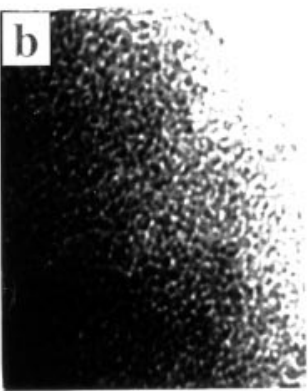

$300 \AA$

Fig. 2 Typical TEM images of as-synthesized and mesoporous ICMUV-3 solids having a P/Al molar ratio of (a) 0.17 and (b) 0.74

peak appears at lower $d$-spacing as the P/Al ratio increases. Additionally, as-synthesized ICMUV-3 materials with high phosphorus content $(\mathrm{P} / \mathrm{Al}>0.4)$ show XRD peaks that can be assigned to the (110) and (200) reflections assuming an hexagonal MCM-41-type cell. 5 When comparing as-synthesized solids (mesostructured) with the corresponding mesoporous materials, we can see that calcination results in an intensity decrease and a broadening of the XRD peak together with a significant shift towards higher $2 \theta$ values. In general, these effects increase as the phosphorus content in the solids does.

Representative TEM images of both as-synthesized and mesoporous ICMUV-3 materials are shown in Fig. 2. On the lines of the XRD observations, it can be noted that while the assynthesized solids show hexagonal (though disordered) pore distributions, the pore packing motif in the mesoporous materials can be better described as wormhole-like.

All ICMUV-3 aluminophosphates show one well defined step in their $\mathrm{N}_{2}$ adsorption isotherm. Two different behaviours are, however, observed: while solids with low $\mathrm{P} / \mathrm{Al}$ ratio present type IV isotherms, materials with high $\mathrm{P} / \mathrm{Al}$ ratio show type I behaviour. The presence of hysteresis loops in the type IV desorption isotherms, as well as their sharp curvature, suggests the existence of a certain blocking in the pore system of the solids with lower P/Al molar ratio. Similar isotherm curves have been previously observed in aluminas prepared through a related surfactant-assisted procedure. ${ }^{14,15}$ As can be seen in Fig. 3 , average pore diameters (BJH model) for ICMUV-3 solids seem to agree with XRD data, and a continuous pore size modulation in the 13-37 A range has been observed. From these data, an almost constant pore wall dimension of $16 \AA$ for all ICMUV-3 solids is obtained.

Besides the pore size adjustment, it is possible to achieve a second and additional tuning by the sole adjustment of the $\mathrm{H}_{2} \mathrm{O}$ / TEA molar ratio in the mother-solution, in a similar way we have previously described for pure aluminas. ${ }^{14}$ Indeed, for a fixed P/Al molar ratio, such pore size modulation allows us to modify the pore dimensions in a range of $c a . \pm 3 \AA$ by adjusting the $\mathrm{H}_{2} \mathrm{O}$ /TEA molar ratio from 34 to 49 .

Hence, the synthetic procedure here described has allowed us to obtain porous aluminophosphates with continuously adjustable pore sizes ranging from micro- $\left(13 \AA, 480 \mathrm{~m}^{2} \mathrm{~g}^{-1}\right)$ to mesoporous ( $37 \AA, 650 \mathrm{~m}^{2} \mathrm{~g}^{-1}$ ) by simple adjustment of the P/ $\mathrm{Al}$ molar ratio in the initial mixture. Though the modulation in pore size is continuous, two groups of materials can be

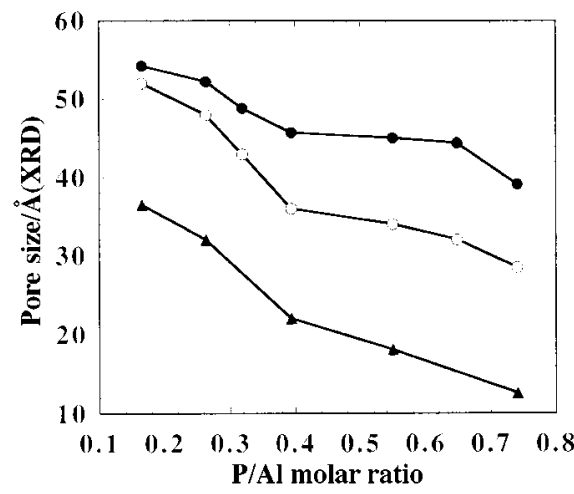

Fig. 3 Evolution of the $d_{100}$ low reflection of both the as-synthesized ( and porous $(\mathrm{O})$ ICMUV-3 and the average pore size $(\boldsymbol{\Delta})$ with the P/Al molar ratio.

differentiated: those with low phosphorus content, whose behaviour is close to that of pure aluminas, and those with high phosphorus content, which likely approach the conventional behaviour of ALPOs. Mesoporous materials with P/Al ratios corresponding to the second group present a more ordered pore distribution.

We conclude that thermally stable micro/mesoporous aluminophosphates with continuously adjustable pore sizes can be prepared in aqueous/TEA through the surfactant assisted procedure using $\mathrm{CTABr}$. Taking into account the ready formation of stable TEA-Al ${ }^{3+}$ complexes, ${ }^{14,16}$ the principal role played by TEA can be postulated as a 'hydrolysis retarding agent'. In this way, its presence in the reaction medium is essential for the isolation of mesostructured ICMUV-3 solids by allowing a satisfactory balance between the two principal processes which take place in solution: the hydrolysis and condensation reactions of aluminium cations in water-phosphoric media and the self-assembling between organic and inorganic moieties.

This research was supported by D. G. E. S. under grant PB951094. J. E. H. and S. C. thank the A. E. C. I. for doctoral grants.

\section{Notes and references}

1 S. T. Wilson, B. M. Lok, C. A. Messina, T. R. Cannon and E. M. Flanigan, J. Am. Chem. Soc., 1982, 104, 1176.

2 M. E. Davis, C. Saldariaga, C. Montes, J. Garces and C. Crowder, Nature, 1988, 331, 698.

3 R. J. Francis and D. O'Hare, J. Chem. Soc., Dalton Trans., 1998, 3133.

4 M. E. Davis and R. F. Lobo, Chem. Mater., 1992, 4, 756

5 C. T. Kresge, M. E. Leonowicz, W. J. Roth, J. C. Vartuli and J. S.Beck, Nature, 1992, 359, 710

6 Q. Huo, D. I. Margolese, U. Ciesla, P. Feng, T. E. Gier, P. Sieger, R. Leon, P. M. Petroff and G. D. Stucky, Nature, 1994, 368, 317.

7 A. Sayari, V. R. Karra, J. S. Reddy and I. L. Moudrakovski, Chem. Commun., 1996, 411.

8 P. Feng, Y. Xia, J. Feng, X. Bu and G. D. Stucky, Chem. Commun., $1997,949$.

9 B. T. Holland, P. K. Isbester, C. F. Blanford, E. J. Munson and A. Stein, J. Am. Chem. Soc., 1997, 119, 6796.

10 D. Zhao, Z. Luan and L. Kevan, Chem. Commun., 1997, 1009.

11 Z. Luan, D. Zhao, H. He, J. Klinowski and L. Kevan, J. Phys. Chem. B, 1998, 102, 1250

12 T. Kimura, Y. Sugahara and K. Kuroda, Chem. Commun., 1998, 559.

13 T. Kimura, Y. Sugahara and K. Kuroda, Microporous Mesoporous Mater., 1998, 22, 115.

14 S. Cabrera, J. El Haskouri, J. Alamo, A. Beltrán, D. Beltrán, S. Mendioroz, M. D. Marcos and P. Amorós, Adv. Mater., in press

15 S. A. Bagshaw and T. J. Pinnavaia, Angew. Chem., Int. Ed. Engl., 1996, 35, 1102 .

16 K. F. Waldner, R. M. Laine, S. Dhumrongvaraporn, S. Tayaniphan and R. Narayanan, Chem. Mater., 1996, 36, 516. 